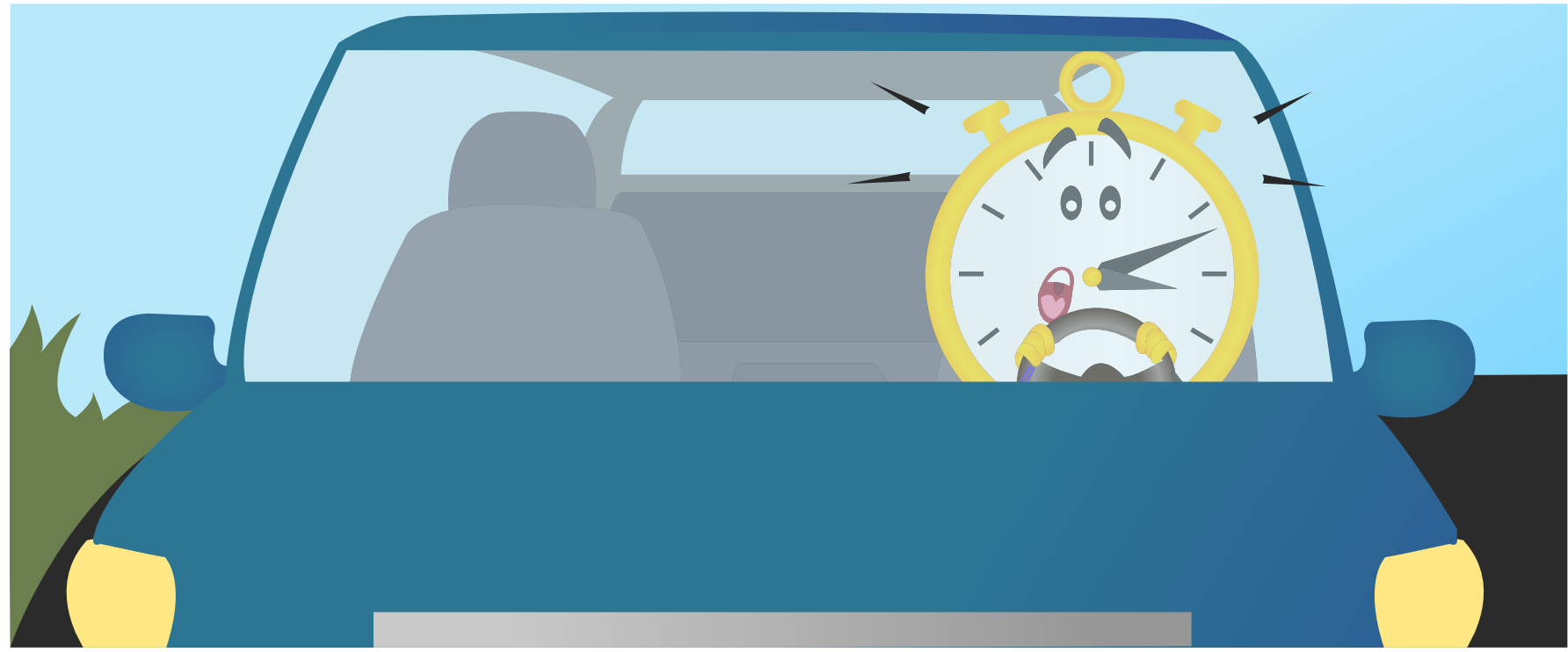

\title{
WHY TIME SLOWS DOWN DURING AN ACCIDENT
}

\section{Marc Wittmann $^{1 *}$ and Virginie van Wassenhove ${ }^{2}$}

${ }^{1}$ Institute for Frontier Areas of Psychology and Mental Health, Freiburg, Germany, ${ }^{2}$ CEA, DSV/I2BM, NeuroSpin Center, Gif-sur-Yvette, France

\section{REVIEWED BY:}

TRAFALGAR SCHOOL FOR GIRLS 14-15 YEARS OLD
After an accident, people often report that it felt a lot longer than it actually could have been in real time. Time seemed to slow down during the event. We tried to conduct a safe experiment in our laboratory to simulate a dangerous situation. We had participants look at a screen on which circles either moved on a collision course toward the participant or moved away from the participant. At the same time, we recorded the participant's brain activation with a brain scanner. When the circle moved toward the participant, that event was judged to last longer than when the circle moved away. This is the effect we wanted to produce: in a situation of "threat," events seem to last longer. The brain activation showed that an area in the middle part of the brain was especially activated, a region that is often active when events have something personal to do with the participant. In our case, a threatening stimulus approaching is the event that is related to the participant. This is the first study to show the regions of the brain that are associated with a perceived slowing down of time during a threatening situation. 
It happened to me (Marc Wittmann) once: I was driving my car on a street that was still wet from the previous night's rainfall. When I turned a corner, the rear tires lost their grip and skidded away. In that moment, when I knew that I had to steer against the skidding of my car, time slowed down. I was very calm and waited for the right moment when I had to turn the steering wheel. Everything seemed to happen in slow motion. I eventually moved the steering wheel and my car was on track again. The event happened perhaps in a second or two, but it felt much longer. Have you had this kind of special experience yourself? Many people we have talked to reported similar experiences, for example, when they had an accident with a bike, or when they had a fall. Time seemed much longer and the world appeared to be in slow motion.

We all know how time can slow down when we are bored. In contrast, when we are having fun, time seems to fly. However, it is not clock time that changes, but our own personal experience of time with respect to the external world. We know that our personal experience of time is dependent upon how we feel and how we are engaged in activities. When we are waiting for something to happen, we are aware of time and time passes very slowly. During joyful activities, time speeds up and we might be surprised by how much time has passed already. But, the slow-motion effect during accidents is an extreme state of consciousness that needs explanation. How does it happen and why?

\section{FIGHT AND FLIGHT: MORE TIME FOR SURVIVAL}

The analyses of hundreds of reports after accidents revealed that $71 \%$ of people recall experiencing an altered passage of time. They recalled the duration of the event as much longer than it actually was and what was happening during an accident seemed to slow down. Moreover, in these situations, people often find that they are thinking very quickly [1]. Why would this actually happen? The answer many researchers would give is this: in a situation of "fight or flight," when it is important for our survival to act very quickly, it helps if the outside world slows down. It then feels as if we have more time to decide what to do next and to move, if necessary. But, what is really happening is that the bodily processes are speeding up relative to the world outside, which makes us feel as if the outside world is slowing down. The arousal level of the body is heightened to its peak. Arousal means that the body and mind are in a physical and mental state of being highly awake and active. The mind is focused and we feel alert. This bodily situation increases the chance of survival, when we have to defend ourselves or have to quickly run away from danger. Because the body and mind are in a mode of extreme speed, it seems like what is happening outside in the world then slows down. Because everything seems to slow down in the environment, we see and hear more details of what is happening, which in turn leads to the feeling that the event lasts longer than it actually does. 


\section{SIMULATING A THREATENING SITUATION IN THE LABORATORY: OUR FMRI STUDY}

But is the explanation given above actually true? Does time expand when people have an accident? Or is it perhaps only later, when we look back, that we feel the event has lasted longer? Due to the dramatic situation, we might think that time slowed down when we recall the event later. So, the question is: can we investigate this phenomenon in the laboratory to verify whether time really expands? Of course, we cannot arrange a real accident in our lab. But, we can think of an experiment with which we might still be able to measure some change in a person's experience of time. Based on an earlier study conducted by one of us (Virginie, who was then at the University of California Los Angeles), we knew that the length of an event feels longer when a stimulus on a screen seems to "loom" toward the viewer [2]. Here is the experimental setup that we used: participants sat in front of a computer screen and saw three filled circles appear, one after the other (see Figure 1 and the movie clip). Then, a fourth circle (the target) appeared on the screen. The participant had to judge how long the fourth circle was visible compared with the three circles appearing before. Did the fourth circle stay on the screen a longer or shorter time than the three previous circles? The fourth circle came in three ways. Either it was another circle that stayed the same size (steady condition), or it loomed (a small circle got bigger and bigger), or it receded (a big circle got smaller and smaller). After another a fifth circle appeared, participants had to

\section{FIGURE 1}

Three different conditions presented to the participants with the steady, loom, and receding target as the fourth stimulus in a series of five ( $t 1-t 5)$.

STEADY: the fourth circle does not change/move. LOOM: the fourth circle gets bigger. RECEDE: the fourth circle gets smaller.

The presentation of circles happens in a sequence (from left to right) starting with $\mathrm{t1}$ (time of circle 1) to t5 (time of circle 5). The duration of the circles were the same (half a second). You may notice that the length of time that passed from one circle to the next was not always the same. The reason behind this was that we wanted participants not get used to a fixed rhythm but concentrate on the length of the circles. press one of two buttons to indicate how long the fourth circle stayed visible: was it shorter or longer than the first three? One button was for "longer" and another button was for "shorter."

Why did we have "looming" and "receding" circles, and what does looming and receding have to do with this, anyway? For someone watching the circles, the growing or looming circle seems to get nearer and nearer. In contrast, the shrinking or receding circle seems to move away from the viewer. The looming circle looks like it is approaching the participant, so we hoped that it might appear to be threatening, since it seems to be on a collision course with the viewer. That way, we tried to simulate a situation where the viewer is confronted

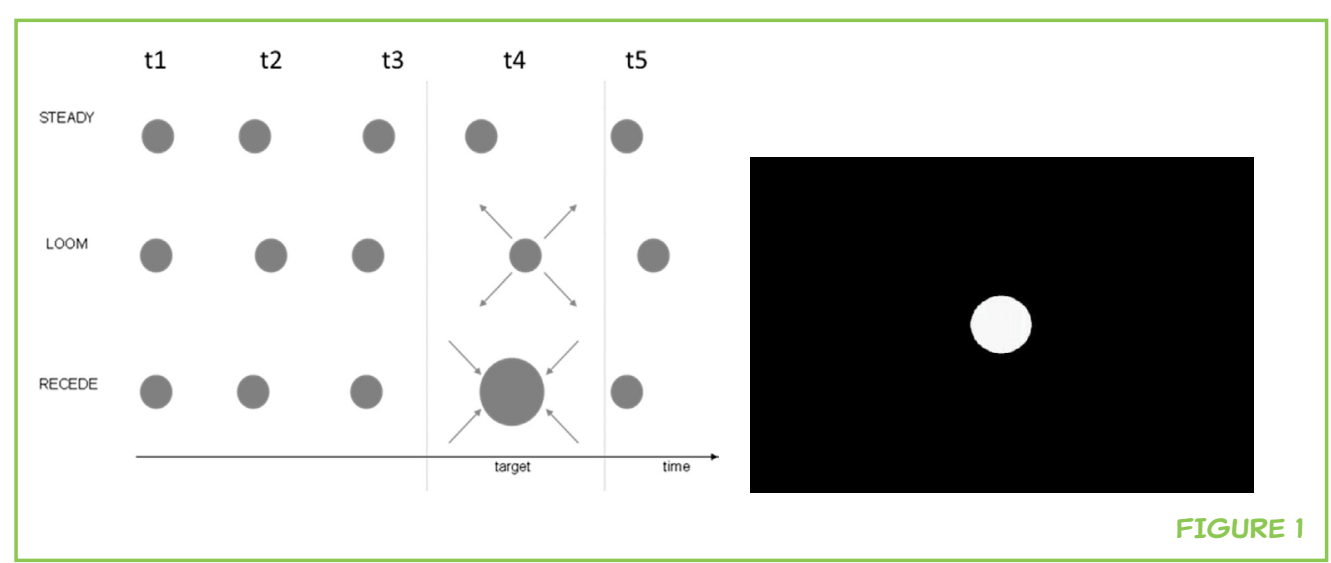


with "danger." We are, of course, aware that this is not a real danger situation. However, the brain might still react as if the stimulation was a minor situation of danger. To make a long story short, the results showed that the looming circle was in fact experienced to last longer than the steady or the receding circles. That is, participants pressed the "longer" button more often than the "shorter" button when they experienced the looming condition. All three types of circles appeared for around half a second. However, the participants stated that the looming circle lasted longer than the two other circles, even when all the circles were actually visible for the same amount of time. So, we found a small but clear effect of time expansion in the situation of a looming, virtually approaching stimulus!

Now the next question is: what is happening in the brain when someone overestimates the amount of time that has gone by, when they feel like the looming circle lasts longer? To answer this question, we used the same computer experiment, but this time the participants were lying in a scanner called a functional magnetic resonance imaging (fMRI) scanner. With the fMRI, we can record activation in the brain while the participant is doing a task. What does "activation" mean? With the fMRI technology, what we actually record are changes in the oxygen levels in the blood of the brain. The idea is that the more a brain region is involved in a task, the more oxygen it needs. When a person is doing a task in the fMRI scanner, we know that more activation in a certain brain region is recorded because this brain region is involved in the task the person is doing. For example, when someone hears sounds, the brain region involved in hearing is active; when someone presses a button, another region of the brain is active that is related to motor action (movement). Using the fMRI method, we asked which brain regions would be active when people see the looming circle as opposed to the receding circle. We measure this activation as "\% signal change" because we are looking at the difference between the two experimental conditions (the looming circle contrasted with the receding circle) and these different conditions result in a difference in oxygen supplied by the blood to a particular brain region. In both conditions (looming and receding circles), something is moving on the computer screen. However, the circle only appears to approach the participant in the looming condition. That is the difference we were interested in.

In this study, we had 15 participants, students from the local University, performing the computer task with the circles while they were in the fMRI scanner. Similar to the results obtained outside the scanner, when performing the task inside the scanner, participants felt like the looming circle lasted longer than the other circles. So, which brain region was active?

In Figure 2, you can see more brain activation in the looming versus the receding condition (in yellow) recorded in two regions of the brain. These regions are both in the cortex, the outermost layer of the brain. One region 


\section{FIGURE 2}

Brain activation for the Loom versus the Recede condition.

The brain areas shown in yellow (numbers 1 and 2) are those that became active in the Loom condition. These are the areas of the brain which are active during the "threat" condition: (1) Cingulate cortex, (2) superior frontal cortex. The areas in blue (numbers 3, 4, and 5) decreased their activity in the Loom condition.

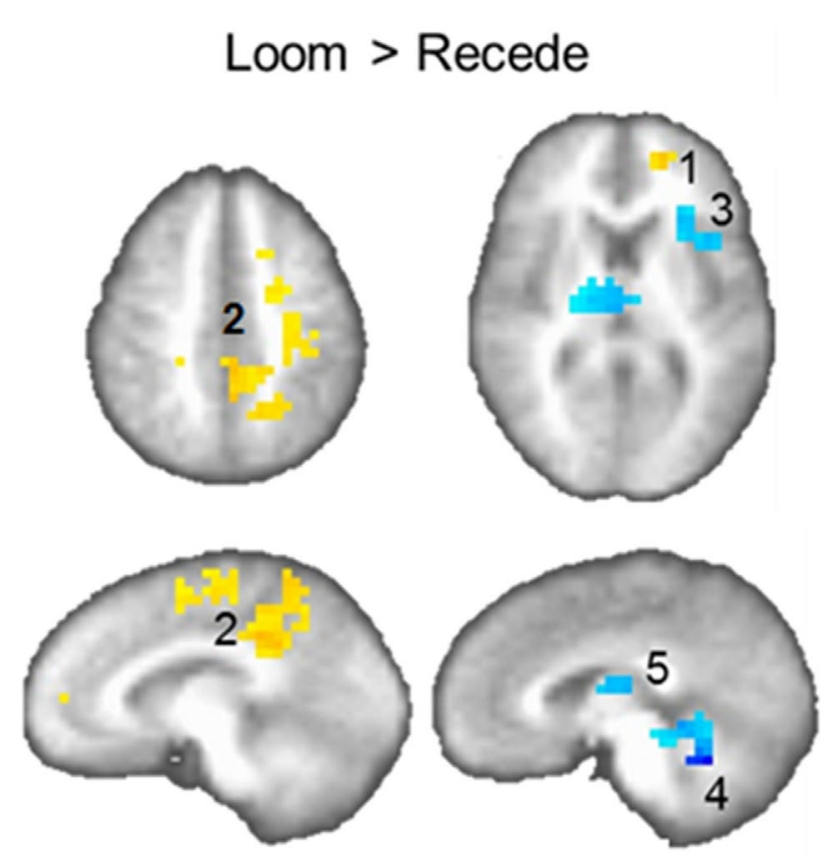

FIGURE 2

of activation was seen in an area called the superior frontal cortex, that is, the outer front part of the cortex. The other region of activation was seen in an area called the medial frontal and posterior cingulate cortex, which means the front and back parts of a region called the cingulate cortex. We have to be very careful in interpreting fMRI data. So keep in mind that the following discussion is only our best idea about what the activation means. We will focus on the larger region of brain activation, the cingulate cortex.

In several studies, it has been shown that the cingulate cortex are active when stimuli have something personal to do with the viewer (to know more about these research findings, see our two articles on the study reported here: Wittmann et al. [3], van Wassenhove et al. [4]). For example, when people think about their own personality traits ("I am a polite person") or when they think about things that they have done ("what a nice party, when I was at last night"), the cingulate cortex is activated. Overall, brain researchers think that the cingulate cortex has a role in people's thinking about himself or herself and thinking about the world and what it has to do with them. In the looming condition, the cingulate cortex becomes activated because the circle moves toward the participant, so the circle has meaning to them because they see it as a potential "threat." He or she might think: It is me who is "threatened." As a result of this threatening situation, the participant's personal sense of time expands.

\section{THE LAB VERSUS THE REAL WORLD}

Our findings were the first to suggest that structures of the brain that are involved in thinking about our relationships with objects in the world may 
also be activated while viewing an approaching object. So, we managed to confirm the result that time expands in a "threatening" situation in an experimental setup in the lab, using the looming circle on the computer screen. One answer to the question of what happens in the brain during this kind of event can be given: activation in the cingulate cortex is recorded with fMRI, which we interpret as the participant thinking about him/herself in relationship to the looming circle. We are, of course, aware that our setup does not come close to an actual accident situation. That is why we can measure a participant's feeling about how much time has gone by, but we could not provoke a real slow-motion effect like people experience during accidents. Real-life situations such as during bungee jumping or parachuting, or an experiment once done by colleagues of ours by having subjects fall into a net from a platform and record how much time they felt passed [5], are more realistic but much harder to perform, and it would be impossible to record brain activity in these situations. But that may be a task done with future technology, where subjects wear helmets with little brain scanners inside and then jump off of a bridge on a rope. Or, researchers could use a virtual reality setting, where events happening to the participant appear highly realistic. But at this point, at least we did conduct a study that brought us a little step closer to understanding what happens in the brain during the slow-motion effect people experience during accidents.

\section{ORIGINAL ARTICLE REFERENCE}

van Wassenhove, V., Wittmann, M., Craig, A. D., and Paulus, M. P. 2011. Psychological and neural mechanisms of subjective time dilation. Front. Neurosci. 5:56. doi:10.3389/fnins.2011.00056

\section{REFERENCES}

1. Arstila, V. 2012. Time slows down during accidents. Front. Psychol. 3:196. doi:10.3389/ fpsyg.2012.00196

2. van Wassenhove, V., Buonomano, D. V., Shimojo, S., and Shams, L. 2008. Distortions of subjective time perception within and across senses. PLOS ONE 3:e1437. doi:10.1371/journal.pone.0001437

3. Wittmann, M., van Wassenhove, V., Craig, B., and Paulus, M. P. 2010. The neural substrates of subjective time dilation. Front. Hum. Neurosci. 4:2. doi:10.3389/ neuro.09.002.2010

4. van Wassenhove, V., Wittmann, M., Craig, A. D., and Paulus, M. P. 2011. Psychological and neural mechanisms of subjective time dilation. Front. Neurosci. 5:56. doi:10.3389/fnins.2011.00056

5. Stetson, C., Fiesta, M. P., and Eagleman, D. M. 2007. Does time really slow down during a frightening event? PLOS ONE 2:e1295. doi:10.1371/journal. pone.0001295 
SUBMITTED: 05 September 2016; ACCEPTED: 16 June 2017;

PUBLISHED ONLINE: 06 July 2017.

EDITED BY: Lesley K Fellows, McGill University, Canada

CITATION: Wittmann M and van Wassenhove V (2017) Why Time Slows Down during an Accident. Front. Young Minds 5:32. doi:10.3389/frym.2017.00032

CONFLICT OF INTEREST STATEMENT: The authors declare that the research was conducted in the absence of any commercial or financial relationships that could be construed as a potential conflict of interest.

COPYRIGHT (C) 2017 Wittmann and van Wassenhove. This is an open-access article distributed under the terms of the Creative Commons Attribution License (CC BY). The use, distribution and reproduction in other forums is permitted, provided the original author(s) or licensor are credited and that the original publication in this journal is cited, in accordance with accepted academic practice. No use, distribution or reproduction is permitted which does not comply with these terms.

\section{REVIEWED BY}

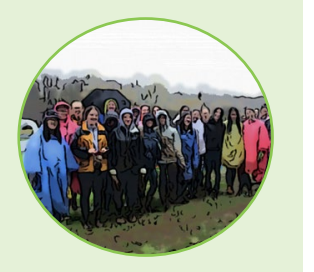

\section{TRAFALGAR SCHOOL FOR GIRLS, 14-15 YEARS OLD}

Trafalgar School for Girls is in the heart of downtown Montreal, QC, Canada. Students in the secondary III science class are learning about human biology and anatomy this year, so helping out with this article was a perfect fit for the curriculum!

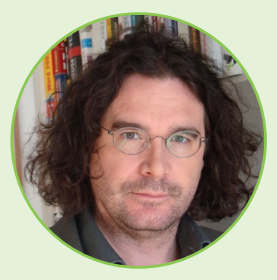

\section{AUTHORS}

\section{MARC WITTMANN}

I am research fellow at the Institute for Frontier Areas of Psychology and Mental Health in Freiburg, Germany. My main research interest lies in how we perceive time. Sometimes time flies by so quickly, but at some other times it just does not want to pass. Why is that so and what mechanisms in the brain are related to these time experiences? Extreme cases of time distortions in extreme states of consciousness, such as when time seems to stand still, are particularly interesting for me. In my spare time I like to read a lot, from novels to science books. Always curious about life, the mind and how people connect. *wittmann@igpp.de

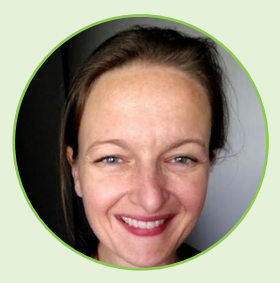

\section{VIRGINIE VAN WASSENHOVE}

I am a cognitive neuroscientist studying the human mind with neuroimaging tools. I lead a team of passionate scientists at the Comissariat à l'Energie Atomique et aux Energies Alternatives (CEA) and at the National Institute of Health and Medical Research (INSERM) in France. My main research questions are: why is it that humans, of all animals, can not only perceive time but also speak about it and imagine a future that does not yet exist? And how is it that our brain, despite all its computing and information processing delays, manages to make our mental world make sense with the outside world? In my spare time, I enjoy listening to and playing music, reading, sporting and all human activities that play with time. 